\title{
Peripheral BDNF and psycho-behavioral aspects are positively modulated by high-intensity intermittent exercise and fitness in healthy women
}

\section{Rodrigo de Poli}

Laboratory of Physiology and Sport Performance (LAFIDE), Post-Graduate Program in Movement Sciences, São Paulo State University (UNESP), School of Science

\section{Vithor Lopes}

Laboratory of Physiology and Sport Performance (LAFIDE), Post-Graduate Program in Movement Sciences, São Paulo State University (UNESP), School of Science

\section{Fabio Lira}

Exercise and Immunometabolism Research Group, Post-Graduation Program in Movement Sciences, Department of Physical Education, São Paulo State University (UNESP)

\section{Alessandro Zagatto}

Laboratory of Physiology and Sport Performance (LAFIDE), Post-Graduate Program in Movement Sciences, São Paulo State University (UNESP), School of Science

\section{Alberto Jimenez-Maldonado}

Facultad de Deportes Campus Ensenada, Universidad Autónoma de Baja California

\section{Barbara Antunes ( $\sim$ ba.antunes2@gmail.com )}

Laboratory of Physiology and Sport Performance (LAFIDE), Post-Graduate Program in Movement Sciences, São Paulo State University (UNESP), School of Science

\section{Research Article}

Keywords: High intensity interval training, menstrual cycle, brain derived neurotrophic factor, cognitive function, psycho-behavioral

Posted Date: December 10th, 2020

DOl: https://doi.org/10.21203/rs.3.rs-119616/v1

License: (c) (i) This work is licensed under a Creative Commons Attribution 4.0 International License. Read Full License

Version of Record: A version of this preprint was published at Scientific Reports on February 18th, 2021. See the published version at https://doi.org/10.1038/s41598-021-83072-9. 



\title{
Peripheral BDNF and psycho-behavioral aspects are positively modulated by high- intensity intermittent exercise and fitness in healthy women
}

\begin{abstract}
Rodrigo Araujo Bonetti de Poli ${ }^{1}$, Vithor Hugo Fialho Lopes ${ }^{1}$, Fábio dos Santos Lira ${ }^{2}$, Alessandro Moura Zagatto ${ }^{1}$, Alberto Jimenez Maldonado ${ }^{3}$, Barbara Moura Antunes ${ }^{* 1,2}$.

1 - Laboratory of Physiology and Sport Performance (LAFIDE), Post-Graduate Program in Movement Sciences, São Paulo State University (UNESP), School of Science. Bauru, SP, Brazil.

2 - Exercise and Immunometabolism Research Group, Post-Graduation Program in Movement Sciences, Department of Physical Education, São Paulo State University (UNESP), Presidente Prudente, SP, Brazil. 3 - Facultad de Deportes Campus Ensenada, Universidad Autónoma de Baja California, México.
\end{abstract}

\section{*Corresponding author:}

PhD Barbara Moura Antunes,

Laboratory of Physiology and Sport Performance (LAFIDE); Department of Physical Education, School of Sciences, São Paulo State University (UNESP), Av. Eng. Luiz Edmundo Carrijo Coube, 14-01, Vargem Limpa, CEP 17033-360, Bauru, SP, Brazil. Phone/Fax: +55 1431036082 Branch Line: 9620.

e-mail: ba.antunes2@gmail.com. 


\begin{abstract}
The study aimed to investigate the effect of high-intensity intermittent effort (HIIE) performed in luteal and follicular menstrual phases on BDNF, cognitive function, mood and exercise enjoyment. Fourteen healthy women completed four experimental sessions, randomly. For each menstrual phase one graded exercise test (GXT) and one HIIE session (10×1-min runs $90 \%$ peak GXT velocity [1-min recovery]) were performed. Blood samples were collected at rest and immediately after efforts, as well profile of mood states questionnaire (POMS) and Stroop-task application. During the HIIE, subjective scales were applied (feeling, felt arousal, rate of perceived exertion and physical activity enjoyment). In serum BDNF no difference was observed between menstrual phases ( $p$ $=0.87)$. Nevertheless, HIIE increased BDNF concentration during two phases $(p=0.03)$. In addition, the magnitude of circulating BDNF changes (BDNF $\Delta$ ) and $\mathrm{VO}_{2 \text { max }}$ showed an inverse relationship in follicular phase $(\mathrm{r}=-0.539, p=0.046)$, whereas in luteal phase the BDNF $\Delta$ were negatively correlated with duration $(r=-0.684, p=0.007)$ and $\mathrm{RPE}(\mathrm{r}=-$ 0.726, $p=0.004)$ in GXT. No difference between menstrual phases was observed for POMS ( $p \geq 0.05)$; however, HIIE attenuates tension $(p<0.01)$, depression $(p<0.01)$ and anger moods $(p<0.01)$, independently of menstrual phases. Subjective scales and Strooptask did not show differences. Menstrual cycle does not affect BDNF, cognitive function, mood and exercise enjoyment, but HIIE increases peripheral BDNF and attenuate tension, depression and anger independently of menstrual phase. Besides, the BDNF $\Delta$ were correlated with fitness status in follicular phase, exhibiting higher changes in women with lower fitness status.
\end{abstract}

Key words: High intensity interval training; menstrual cycle; brain derived neurotrophic factor; cognitive function; psycho-behavioral 


\section{Introduction}

The physical exercise activates several metabolic pathways in the skeletal muscle, and regulate the synthesis and secretion of molecules known as myokines ${ }^{1-4}$. The myokines have autocrine, paracrine or endocrine effects ${ }^{5}$. Therefore, through myokines, the skeletal muscle generates a crosstalk with other organs such as adipose tissue, liver and brain ${ }^{6,7}$. In detail, Cathepsin-b and Irisin were identified as myokines that enhance the brain-derived neurotrophic factor (BDNF) expression in brain ${ }^{8}$, effect that was found even after a single bout of high-intensity intermittent effort (HIIE) ${ }^{8}$. In agreement with this, other authors reported higher peripheral BDNF levels after HIIE ${ }^{9,10}$, The BDNF is a neurothrophin that regulate molecular pathways associated to neurogenesis, synaptic plasticity and metabolism in neurons ${ }^{11}$. The prior information emphasizes the potential of intermittent exercise modality to promote brain health. In fact, it is known that the physical exercise arouse dopamine pathways and consequently act on neurobehavioral systems, influencing positively the mood, exercise enjoyment and perceived ${ }^{12}$.

However, besides the physical exercise, there are another factors that modify BDNF concentrations, one of them are the ovarian hormones ${ }^{13}$. In the light of this, Begliuomini et al ${ }^{14}$ reported higher BDNF concentrations during luteal phase than follicular phase; the same study found a positive relationship between BDNF and progesterone concentrations (i.e., hormone expressed during luteal phase). These data suggested that progesterone impact directly on brain (i.e., central nervous system) modifying the BDNF concentration. In fact, was previously reported that ovarian hormones alter different brain functions such as cognition, memory and mood ${ }^{15}$.

Although the previous studies demonstrated the influence of menstrual cycle on BDNF, the impact of ovarian hormones on HIIE responses and the relationship between physical performance and BDNF remain unclear. Therefore, the principal aim of the current study was to evaluate the effect of HIIE performed in the luteal and follicular menstrual cycle phases on BDNF concentrations, cognitive function, mood and exercise enjoyment in healthy women.

\section{Results}

The parameter measured on GXT and comparisons between luteal and follicular phases are showing in Supplemental Table S1. No difference was observed for any parameter measured on GXT between menstrual phases $(p \geq 0.13)$. 
Regarding the results of the mood states profile measured previously and immediately after HIIE (Table 1), no significant interaction among time and conditions and for condition effect were observed for all POMS dimensions. Contrary, HIIE mitigate significantly (i.e., ANOVA time effect) tension ( $\left.p=0.003 ; F=14.355 ; \eta^{2}=0.545\right)$, depression $\left(p=0.008 ; F=10.222 ; \eta^{2}=0.460\right)$ and anger $\left(p=0.001 ; F=18.932 ; \eta^{2}=\right.$ 0.612 ) moods. The enjoyment measured by PACES was not different between luteal and follicular phases [68.07 \pm 5.90 arbitrary unit (a.u.) (CI95\% 64.66 to 71.48 a.u.) and 67.36 \pm 6.00 a.u. (CI95\% 63.90 to 70.82 a.u.), respectively; $p=0.72]$.

\section{***TABLE 1 NEAR HERE***}

No significant differences between time within luteal and follicular phases were observed for RPE $\left(p=0.619 ; F=0.652 ; \eta^{2}=0.048\right)$, pain $\left(p=0.395 ; F=1.023 ; \eta^{2}=\right.$ $0.073)$, feeling $\left(p=0.158 F=1.742 ; \eta^{2}=0.118\right)$, arousal $\left(p=0.324 ; F=1.192 ; \eta^{2}=0.084\right.$ ) and HR ( $p=0.840 ; F=0.543 ; \eta^{2}: 0.043$ ) during the 10 sprints runs of HIIE (Figure 1A to $1 \mathrm{E})$. Likewise, [BLa] after HIIE was not different between luteal and follicular phases $\left[2.17 \pm 0.97 \mathrm{mmol} \cdot \mathrm{L}^{-1}\left(\mathrm{CI} 95 \% 1.61\right.\right.$ to $\left.2.74 \mathrm{mmol} \cdot \mathrm{L}^{-1}\right)$ and $2.23 \pm 1.02 \mathrm{mmol} \cdot \mathrm{L}^{-1}(\mathrm{CI} 95 \%$ 1.64 to $\left.\left.2.82 \mathrm{mmol} \cdot \mathrm{L}^{-1}\right) ; p=0.80\right)$.

\section{*** FIGURE 1 NEAR HERE***}

The time interference score of Stroop test was $11.06 \pm 5.93$ a.u. (CI95\% 7.47 to 14.64 a.u.) and $9.71 \pm 3.66$ a.u. (CI95\% 7.50 to 11.92 a.u.) for pre and post HIIE during luteal phase, while $10.81 \pm 5.70$ a.u. (CI95\% 7.37 to 14.26 a.u.) and $9.86 \pm 6.51$ a.u. (5.92 to 13.79 a.u.) for pre and post-HIIE during follicular phase. There was not significant difference between time within conditions ( $\left.p=0.828 ; F=0.049: \eta^{2}: 0.004\right)$, moments $\left(p=0.207 ; F=1.780 ; \eta^{2}: 0.129\right)$ and menstrual phases $\left(p=0.970 ; F=0.001 ; \eta^{2}: 0.00\right)$.

The Figure 2 showed the BDNF results. Not statistical difference was observed for BDNF absolute values among condition $\left(p=0.87 ; F=0.028 ; \eta^{2}=0.002\right)$ and between pre and post variation $(\Delta \%)$ of each phase. In addition, no difference between menstrual phases were observed $\left(p=0.35 ; F=0.923 ; \eta^{2}=0.066\right)$. On the other hand, a statistical significance was observed between moments $\left(p=0.03 ; F=6.057 ; \eta^{2}=0.318\right)$, showing that BDNF increases significantly after HIIE sessions for both conditions. Delta absolute difference $(\Delta)$ between pre and post HIIE during luteal phase is $8.22 \pm 17.43 \mathrm{ng} / \mathrm{mL}$ (CI95\% -1.85 to $18.28 \mathrm{ng} / \mathrm{mL}$ ) and $7.29 \pm 13.67 \mathrm{ng} / \mathrm{mL}$ (CI95\% -0.61 to $15.19 \mathrm{ng} / \mathrm{mL}$ ). Similarly, $\Delta \%$, the delta absolute difference was not different between conditions $(p=$ $0.87)$. 
Correlation between $\triangle \% \mathrm{BDNF}$ and $\dot{\mathrm{VO}}_{2 \max }$, GXT performance and RPE were expressed in Figure 3. For follicular phase, only $\dot{\mathrm{VO}}_{2 \max }$ showed a significant correlation with $\Delta \% \operatorname{BDNF}(\mathrm{r}=-0.539 ; p=0.046)$, while in luteal phase a significant correlation was observed between $\Delta \%$ BDNF and GXT time to exhaustion $(\mathrm{r}=-0.684 ; p=0.007)$ and RPE $(r=-0.726 ; p=0.004)$. Opposite, non-significant correlation was observed between $\Delta \%$ BDNF and $i \dot{\mathrm{VO}}_{2 \max }, \mathrm{PI}-\mathrm{GXT}$ and [BLa] $]_{\text {peak }}$ for both phases $(\mathrm{r} \geq-0.51 ; p \geq 0.06)$. In addition, were not correlation between $\triangle \%$ BDNF and POMS dimensions of moods for luteal ( $\mathrm{r} \geq-0.254 ; p \geq 0.14$ ) and follicular phase ( $\mathrm{r} \geq-0.247 ; p \geq 0.14)$, as well with any subjective measurements and with peak of [BLa] after HIIE for both phases $(r \geq-0.378$; $p \geq 0.25)$.

Finally, any significant correlation was found among $\Delta \%$ BDNF and time interference score of Stroop test for luteal $(\mathrm{r} \geq 0.285 ; p \geq 0.346)$ and follicular phase $(\mathrm{r} \geq$ $-0.273 ; p \geq 0.368)$.

***FIGURE 3 NEAR HERE***

\section{Discussion}

The current study was focused to assess the impact of HIIE performed in the luteal and follicular menstrual cycle phases on BDNF concentrations, cognitive function, mood and exercise enjoyment in healthy women. Here, we showed for the first time that independently of menstrual phase, HIIE increases the circulating BDNF levels; additionally, data of the current work indicate that HIIE mitigates the tension, depression and anger measured with POMS. On the other hand, we identified a negative relationship between the magnitude of peripheral BDNF changes (post-pre HIIE session) and $\dot{\mathrm{VO}}_{2 \max }$ in follicular phase. In the luteal phase an inverse relationship among BDNF changes (post-pre HIIE session) with GTX-time and GTX-RPE were found. These data suggested that the fitness level can exert an important impact on BDNF concentration after HIIE session, mainly during follicular phase.

The non-difference in serum BDNF concentration between luteal and follicular phases are concordant with other authors ${ }^{16}$. In their study, the authors reported any difference between serum BDNF measured prior exercise in luteal and follicular phases, as well when compared with men serum BDNF concentrations ${ }^{16}$. To our best knowledge, there are not more studies focused to assess the impact of HIIE on serum BDNF concentrations during different menstrual phases. Nevertheless, others works have 
identified the role of fitness level to modify, the circulating BDNF concentration after exercise, especially as response to high intensity efforts $9,10,17$, that evidences are concordant with our data, this means a higher serum BDNF concentration after high intensity efforts.

Interestingly, our data indicated a strong relationship between circulating BDNF changes and fitness status in the both menstrual phase cycle. Specifically, a negative correlation between BDNF variation and $\mathrm{VO}_{2 \max }$ was found during the follicular phase; likewise, in luteal phase negative correlation between BDNF variation with GTX-time and GXT-RPE was showed. Despite the menstrual cycle per se does not affect the physical performance ${ }^{18}$, authors reported that the hormonal fluctuation, such as estrogens (i.e., higher levels during follicular phase and lower levels in luteal phase) and progesterone (i.e., lower levels during follicular phase and higher levels during luteal phase) impact on the inflammatory responses ${ }^{19}$. Specifically, the estrogens have an important role to regulate the immunocompetence (i.e., less anti-inflammatory gene activation during luteal phase) ${ }^{19}$. We consider that the last condition could affect the circulating BDNF levels in females. In fact, Weaver et $\mathrm{al}^{20}$ found one association between BDNF and progesterone $\left(r^{2}=0.623, p=0.01\right)$, suggesting that hormone may regulates the BDNF concentration.

Moreover, it suggested that BDNF concentration may be related with lactate accumulation, once is inferred that muscle lactate is capable to crosses the blood-brain barrier and induces the BDNF increase via activation of Sirtuin1 deacetylase (SIRT1) with consequent increases of peroxisome proliferator-activated receptor gamma coactivator-1 alpha (PGC1 $\alpha$ ) and the secreted molecule FNDC5 (myokine gene that mediate BDNF release) ${ }^{21}$; however, there is no correlation between blood lactate concentration after HIIE and serum BDNF (Follicular phase: $\mathrm{r}^{2}=-0.24, p=0.40$; Luteal phase: $\left.\mathrm{r}^{2}=0.05 ; p=0.85\right)$. One possible explanation for this result is the measurement of blood lactate concentration after HIIE, once the passive intervals between the sprints may underestimate the real concentration of the 10 high intensity efforts of HIIT ${ }^{22}$.

Besides the putative effect on BDNF, the ovarian hormones can act on hypothalamus and modify the mood, pain perception and exercise enjoyment ${ }^{23}$. In regarding with this, no statistical differences were observed for these subjective parameters between menstrual phases (Table 1). However, attenuation of subjective psychological parameters, such as tension, depression and anger state of mood (i.e., POMS) were found in both menstrual phases (Table 1). It is worthy indicate that mood 
variables were not correlated with BDNF concentrations after HIIE, these data suggest that acute mood changes induced by HIIE comes from another factors, such as, the dopamine pathways ${ }^{12}$, once exercise regulation can increase dopamine receptor 2 and 4 sensitivity on hippocampus and this may facilitate dopamine transmission for ventral striatum and nucleus accumbens (brain area related with exercise motivation and behavioral processes) ${ }^{24}$; However, this pathway was not measured in the present study. Further studies are justify to elucidate the possible impact of HIIE on dopamine system.

It is worth indicate some precautions must be consider during the interpretation of the presented data, mainly that even as to all participants should have a regular menstrual cycle, the luteal and follicular phase were defined by self-reported control methods, without the hormonal dosage ${ }^{18}$. In this way, is possible that participants with the symptoms of menstrual cycle but without ovarian hormones fluctuation may has been included in analysis being an important limitation of the current study.

Taken together, our data demonstrate that, menstrual cycle did not impact on serum BDNF concentration, cognitive function, mood and exercise enjoyment in healthy women. However, acute HIIE increases the serum BDNF concentration and attenuate tension, depression and anger state of mood independently of menstrual phase, suggesting the possibility to use HIIE as a treatment to attenuate the deleterious sensations occasioned by ovarian hormonal fluctuations. Even, was observed important relationship between BDNF changes with physical fitness status (i.e., $\dot{\mathrm{VO}}_{2 \max }$ ) in follicular phase, but not in luteal phase. Finally, the study showed that the fitness status may be a relevant factor to regulate the magnitude of circulating BDNF changes during intermittent exercise, this information can be relevant for coaches and trainers.

\section{Methods}

\section{Participants}

A total of 14 healthy and physically active woman volunteers had participated in the study (age: $24 \pm 2$ years; BMI: $22.79 \pm 1.89 \mathrm{~kg} \cdot \mathrm{m}^{2}$ ). Before the begin of experimental procedures, participants were informed about the possible risks and benefits of the study and signing an informed written consent. All procedures were approved by Sao Paulo State University Research Ethics Board (Protocol CAAE 92380318.5.0000.5402) and were conducted according to the Declaration of Helsinki. Additionally, the participants were instructed to not perform any strenuous effort for at least 72 hours between every session and not consume any ergogenic aid (i.e., caffeine) or alcohol drinks. All 
participants should have a regular menstrual cycle, attested by self-reported control methods, and do not use any hormonal contraceptive methods at least 3 months prior the begging of the experiments.

\section{Experimental Design}

Prior the beginning of the experimental procedures, the participants have reported individually their menstrual cycle calendar to identification of the follicular and luteal phases. After this, the participants visited the laboratory for 4 times. 2 visits on follicular phase and 2 on luteal phase, were realized randomly. On first session of each phase the participants underwent to a graded exercise test (GXT) to measure maximal oxygen uptake $\left(\dot{\mathrm{VO}}_{2 \max }\right)$ and the peak intensity reached in GXT (PI-GXT), whereas on second session of each phase, a HIIE was performed at $90 \%$ of PI-GXT. An interval of $72 \mathrm{~h}$ was respected between GXT and HIIE sessions (Supplemental Figure S1).

Ninety minutes before the HIIE sessions, a standard breakfast was offered to all participants (equivalent to $30 \%$ of individually estimated daily caloric expenditure) with macronutrient distribution of $\approx 35 \%$ lipids, $\approx 50 \%$ carbohydrates and $\approx 15 \%$ proteins ${ }^{25}$. Before (90 minutes after breakfast) and immediately after HIIE the inhibitory cognitive control was assessed by Stroop color word test (SCWT), while and mood state was assessed by Profile of Mood States Questionnaire (POMS), respectively. Venous blood sample was collected before and immediately for BDNF measurement. During every HIIE and after the end of each session, pleasure/displeasure, pain perception, arousal level and rate of perceived exertion was assessed. After HIIE, the exercise enjoyment was measured by Physical Activity Enjoyment Scale. In addition, 3 and 5 minutes after GXT and HIIE sessions capillarized blood samples of ear lobe were collected to determine peak of blood lactate concentrations ([La $\left.]_{\text {peak }}\right)$.

All exercise sessions were performed on a motorized treadmill (ATL, Inbramed, Porto Alegre, Brazil) with a fixed gradient at $1 \%{ }^{26}$, using a safety belt to avoid accidental falls and to induce the maximal performance.

\section{Graded Exercise Test}

The GXT started at $6.5 \mathrm{~km} \cdot \mathrm{h}^{-1}$ with $1.5 \mathrm{~km} \cdot \mathrm{h}^{-1}$ of increment every 2 minutes until the voluntary exhaustion ${ }^{27}$. Immediately exhaustion, the participants remained in passive recovery during 5 minutes and performed a supramaximal effort until exhaustion at intensity of $105 \%$ of the PI-GXT (verification test) ${ }^{28}$. This supramaximal effort was used 
as a $\dot{\mathrm{V}}_{2 \max }$ confirmation. During GXT and supramaximal effort (i.e., confirmation of $\dot{\mathrm{V}} \mathrm{O}_{2 \max }$ ), the gas-exchange response was measured breath-by-breath using a gas analyzer (Quark CPET, COSMED, Rome, Italy) previously calibrated according manufacturer's instructions. The heart rate (HR) was also measured using a wireless chest belt (HR Monitor; COSMED, Rome, Italy) synchronized with the gas analyzer. Data were smoothed every 10 points on Omnia Software 1.6.5 (COSMED, Rome, Italy) and interpolated each second with OriginPro 8.0 (OriginLab Corporation, Northampton, Massachusetts, USA).

The oxygen uptake of every completed stage during GXT was determined as mean of last 30 seconds, while the oxygen uptake during supramaximal effort was determined considering the last 15 seconds of the effort ${ }^{28}$. The $\dot{\mathrm{V}} \mathrm{O}_{2 \max }$ was assumed as highest average of oxygen uptake when was observed an oxygen uptake plateau (difference $<2.1$ $\mathrm{ml} \cdot \mathrm{kg}^{-1} \cdot \mathrm{min}^{-1}$ between on oxygen uptake on last two completed stages of GXT). When plateau was not observed, the $\dot{\mathrm{V}} \mathrm{O}_{2 \max }$ was assumed as higher value between highest oxygen uptake value measured on GXT and on supramaximal effort, when the difference was $<2.1 \mathrm{ml} \cdot \mathrm{kg}^{-1} \cdot \mathrm{min}^{-1} 29$. In addition, the $i \dot{\mathrm{V} O}{ }_{2 \max }$ was assumed as the lowest GXT intensity in which $\dot{\mathrm{VO}}_{2 \max }$ was reached. If any $\dot{\mathrm{VO}}_{2 \max }$ criteria were reached, the GXT session was repeated at least $48 \mathrm{~h}$ after.

\section{High-intensity intermittent exercise}

Before HIIE, the participants performed 5 minutes of warm-up at $40 \%$ of $i \dot{\mathrm{VO}}_{2 \max }$. The HIIE sessions consist of 10 runs of 1 minute at $90 \%$ of PI-GXT (upper to $85 \%$ of maximal heart rate) with 1 minute of passive recovery [adapted from Little et al (2010)], as recommended by Gibala, Gillen and Percival ${ }^{31}$ to generate adaptations by training (i.e., target intensity is between 80 and $100 \%$ of maximal HR). The HR was measured beat-by-beat during all HIIE (Polar V800, Kempele, Finland). In addition, all HIIE were performed in the same period of the day to minimize the effects of the circadian cycle (between 7:00 a.m. and 12:00 p.m.) with controlled room temperature.

\section{Stroop Color and Word Test}

The Stroop Test were composed by three sets, those constituted by fifty components and a task ${ }^{32}$. The sets components could be a word or a color, which of those were used the pink, blue and green in accordance with an Brazilian and validated Stroop 
Test version ${ }^{33}$. The first set were formed the for fifty names of colors wrote in black ink and the second had fifty colorful blocks, but in the third the names of colors were printed with an incongruous color ink, those sets were denominated word (W), colors (C) and color word $(\mathrm{CW})$ respectively. In both, word and colors set, the task was to point the components. However, in the color word set, the task was to name the ink color ignoring the word wrote. In all sets, the volunteers were always encouraged to complete the task as faster is possible and the time demanded to complete each task was measured by a chronometer. Moreover, the answers were recorded by a voice record. After this, the time interference score (Ti) was calculated using the equation 1 (Eq.1 ${ }^{34}$.

$$
\text { Eq.1 Ti }=\text { CWT- }\left[\frac{(\text { WT-CT })}{2}\right]
$$

Where CWT, WT and CT refers to time to complete the task in color word, word and color trial respectively.

\section{Subjective perceptions measurements}

All participants were familiarized with scales and questionnaires before the HIIE sessions. The mood state was assessed using the profile of mood states questionnaire (POMS) ${ }^{35}$. The scores of tension, depression, anger, vigor, fatigue and confusion was measured according Raglin and Morgan ${ }^{35}$. The pleasure/displeasure was assessed using the Feeling Scale ${ }^{36}$, while legs pain perception was assessed by Pain Intensity Scale (0 to 10$)^{37}$, arousal level by Felt Arousal Scale (0 to 6) ${ }^{38}$ and rate of perceived exertion (RPE) by Borg Scale (6-20) ${ }^{39}$. Enjoyment assessed after HIIE was measured by original 18 items Physical Activity Enjoyment Scale (PACES) with 7 points bipolar ratings ${ }^{40,41}$. Values of each item were sum, being the minimum score 18 points and 126 points the maximum score. Higher scores evidenced higher exercise enjoyment ${ }^{41}$.

\section{Blood samples analysis}

Venous blood samples were collected from antecubital vein $(\sim 10 \mathrm{~mL})$ and immediately allocated into vacutainer tubes contained EDTA or anticoagulant for plasma and serum separation. The vacutainer tubes were centrifuged for 15 minutes at $3000 \mathrm{rpm}$ and $4^{\circ} \mathrm{C}$ (Centrifuge $5430 \mathrm{R}$, Eppendorf, Hamburg, Germany) for plasma and serum separation. Next, the plasma and serum content were stored at $-20^{\circ} \mathrm{C}$ until freezes and stored at $-80^{\circ} \mathrm{C}$ until analysis. The serum BDNF concentration was analysed using an 
enzyme-linked immunosorbent assay (ELISA) (R\&D System, Minneapolis, MN, USA). The assay was performed following the to manufacturer's guidelines. Sensibilities of the enzymatic kit was $1500-23.4 \mathrm{pg} / \mathrm{mL}$ with intra-assay coefficient of variation (CV) of $1.3 \%$.

\section{Lactate determination}

The capillary blood was collected from earlobe $(25 \mu \mathrm{L})$ for measurement of blood lactate concentration [BLa]. The blood was collected using heparinized capillaries and stored at $-20^{\circ} \mathrm{C}$ into microtube containing $50 \mu \mathrm{L}$ of $1 \%$ sodium fluoride. The capillary blood samples were analyzed with a biochemical analyzer (YSI 2900; Yellow Spring Instruments, Yellow Spring, Ohio, USA, EUA), with equipment error of $\pm 2 \%$, according the manufacturer information.

\section{Statistical Analysis}

Data are presented as mean \pm SD and 95\% confidence intervals (CI95\%). For metabolic values the percentage of variation $(\Delta \%)$ between menstrual phases and moments (pre and post HIIE) was calculated assuming, as Luteal phase and pre exercise as $100 \%$. ShapiroWilk test was used to verify the normality distribution of data. For comparison between groups (i.e., menstrual cycle phase) and moments (i.e., pre and post HIIE) a two-way analysis of variance for repeated measures (two-way ANOVA) was used. In addition, Mauchly's sphericity test was applied, and sphericity was assumed to be violated when the F-test was significant. In case of sphericity violation, the Greenhouse-Geisser Epsilon correction was used. Effect sizes for the ANOVA were calculated using partial eta squared $\left(\eta^{2}\right)$ for group, time, and interaction. The T-Test was used to compare the $\Delta \%$ between phases and between moments. The Spearman correlation test was used to verify possible association between BDNF, subjective perceptions measurements and Stroop test, while Pearson's correlations was used to verify the possible association between BDNF and blood lactate concentration. In all cases, the significance level was $\leq 5 \%$ ( $\leq$ 0.05) and the data were analyzed using the Statistical Package for Social Sciences 22.0 (SPSS Inc. Chicago. IL.USA).

\section{References}

1. Pedersen, B. K., Åkerström, T. C. A., Nielsen, A. R. \& Fischer, C. P. Role of myokines in exercise and metabolism. J. Appl. Physiol. 103, 1093-1098 (2007). 
2. Febbraio, M. A. \& Pedersen, B. K. Muscle-derived interleukin-6: mechanisms for activation and possible biological roles. FASEB J. 16, 1335-1347 (2002).

3. Steensberg, A. et al. Production of interleukin-6 in contracting human skeletal muscles can account for the exercise-induced increase in plasma interleukin-6. J. Physiol. 529, 237-242 (2000).

4. Steensberg, A. et al. IL-6 and TNF- $\alpha$ expression in, and release from, contracting human skeletal muscle. Am. J. Physiol. - Endocrinol. Metab. 283, E1272-8 (2002).

5. Pedersen, B. K. Muscles and their myokines. J. Exp. Biol. 214, 337-346 (2011).

6. Leal, L. G., Lopes, M. A. \& Batista, M. L. Physical exercise-induced myokines and muscle-adipose tissue crosstalk: A review of current knowledge and the implications for health and metabolic diseases. Front. Physiol. 9, 1307 (2018).

7. Fiuza-Luces, C., Garatachea, N., Berger, N. A. \& Lucia, A. Exercise is the real polypill. Physiology 28, 330-358 (2013).

8. Pedersen, B. K. Physical activity and muscle-brain crosstalk. Nat. Rev. Endocrinol. 15, 383-392 (2019).

9. Cabral-Santos, C. et al. Inflammatory cytokines and BDNF response to highintensity intermittent exercise: Effect the exercise volume. Front. Physiol. 7, 509 (2016).

10. Marquez, C. M. S., Vanaudenaerde, B., Troosters, T. \& Wenderoth, N. Highintensity interval training evokes larger serum BDNF levels compared with intense continuous exercise. J. Appl. Physiol. 119, 1363-1373 (2015).

11. Marosi, K. \& Mattson, M. P. BDNF mediates adaptive brain and body responses to energetic challenges. Trends Endocrinol. Metab. 25, 89-98 (2014).

12. Reed, J. \& Buck, S. The effect of regular aerobic exercise on positive-activated affect: A meta-analysis. Psychol. Sport Exerc. 10, 581-594 (2009).

13. Pluchino, N. et al. Daily variation of brain-derived neurotrophic factor and cortisol in women with normal menstrual cycles, undergoing oral contraception and in postmenopause. Hum. Reprod. 24, 2303-2309 (2009).

14. Begliuomini, S. et al. Influence of endogenous and exogenous sex hormones on plasma brain-derived neurotrophic factor. Hum. Reprod. 22, 995-1002 (2007).

15. McEwen, B. S. \& Milner, T. A. Understanding the broad influence of sex hormones and sex differences in the brain. J. Neurosci. Res. 95, 24-39 (2017).

16. El-Sayes, J. et al. The Effects of Biological Sex and Ovarian Hormones on Exercise-Induced Neuroplasticity. Neuroscience 410, 29-40 (2019). 
17. Antunes, B. M., Rossi, F. E., Teixeira, A. M. \& Lira, F. S. Short-time high-intensity exercise increases peripheral BDNF in a physical fitness-dependent way in healthy men. Eur. J. Sport Sci. 20, 43-50 (2020).

18. Janse De Jonge, X. A. K. Effects of the menstrual cycle on exercise performance. Sport. Med. 33, 833-851 (2003).

19. Abbasi, A. et al. Sex-specific variation in signaling pathways and gene expression patterns in human leukocytes in response to endotoxin and exercise. $J$. Neuroinflammation 13, 289 (2016).

20. Weaver, K. R. et al. Sex Hormones, BDNF, Leptin, and TGF- $\beta 1$ in Females With IBS: A Pilot Investigation. Biol. Res. Nurs. ahead of print, (2020).

21. El Hayek, L. et al. Lactate mediates the effects of exercise on learning and memory through sirt1-dependent activation of hippocampal brain-derived neurotrophic factor (BDNF). J. Neurosci. 39, 2369-2382 (2019).

22. Panissa, V. L. G. et al. Is oxygen uptake measurement enough to estimate energy expenditure during high-intensity intermittent exercise? Quantification of anaerobic contribution by different methods. Front. Physiol. 9, 868 (2018).

23. Schiller, C. E., Johnson, S. L., Abate, A. C., Schmidt, P. J. \& Rubinow, D. R. Reproductive Steroid Regulation of Mood and Behavior. Compr. Physiol. 13, 1135-1160 (2016).

24. Ruegsegger, G. N. \& Booth, F. W. Running from disease: Molecular mechanisms associating dopamine and leptin signaling in the brain with physical inactivity, obesity, and type 2 diabetes. Front. Endocrinol. (Lausanne). 23, :109 (2017).

25. Mifflin, M. D. et al. A new predictive equation for resting energy expenditure in healthy individuals. Am. J. Clin. Nutr. 51, 241-247 (1990).

26. Jones, A. M. \& Doust, J. H. A $1 \%$ treadmill grade most accurately reflects the energetic cost of outdoor running. J. Sports Sci. 14, 321-327 (1996).

27. de Poli, R. de A. B., González, J. A. M., Fonsati, N. \& Zagatto, A. M. Differences between genders in anaerobic capacity during a supramaximal effort. Motriz 25, e101910 (2019).

28. Rossiter, H. B., Kowalchuk, J. M. \& Whipp, B. J. A test to establish maximum O2 uptake despite no plateau in the $\mathrm{O} 2$ uptake response to ramp incremental exercise. J. Appl. Physiol. 100, 764-70 (2006).

29. Schaun, G. Z. The Maximal Oxygen Uptake Verification Phase: a Light at the End of the Tunnel? Sport. Med. Open 3, :44 (2017). 
30. Little, J. P., Safdar, A., Wilkin, G. P., Tarnopolsky, M. A. \& Gibala, M. J. A practical model of low-volume high-intensity interval training induces mitochondrial biogenesis in human skeletal muscle: Potential mechanisms. $J$. Physiol. 588, 1011-1022 (2010).

31. Gibala, M. J., Gillen, J. B. \& Percival, M. E. Physiological and Health-Related Adaptations to Low-Volume Interval Training: Influences of Nutrition and Sex. Sport. Med. 44, 127-137 (2014).

32. Stroop, J. R. Studies of interference in serial verbal reactions. J. Exp. Psychol. 18, 643-662 (1935).

33. Brandelero, V.\& Toni, P. M. De. Study of validity of Stroop Color and Word Test for Inhibitory. Psicol. Argumento 33, 282-297 (2015).

34. Van Der Elst, W., Van Boxtel, M. P. J., Van Breukelen, G. J. P. \& Jolles, J. The stroop color-word test: Influence of age, sex, and education; and normative data for a large sample across the adult age range. Assessment 13, 62-79 (2006).

35. Raglin, J. S. \& Morgan, W. P. Development of a scale for use in monitoring training-induced distress in athletes. Int. J. Sports Med. 15, 84-88 (1994).

36. Hardy, C. J. \& Rejeski, W. J. Not What, but How One Feels: The Measurement of Affect during Exercise. J. Sport Exerc. Psychol. 11, 304-314 (1989).

37. Cook, D. B., O’Connor, P. J., Oliver, S. E. \& Lee, Y. Sex differences in naturally occurring leg muscle pain and exertion during maximal cycle ergometry. Int. J. Neurosci. 95, 183-202 (1998).

38. Svebak, S. \& Murgatroyd, S. Metamotivational Dominance. A Multimethod Validation of Reversal Theory Constructs. J. Pers. Soc. Psychol. 48, 107-116 (1985).

39. Borg, G. A. V. Psychophysical bases of percieved exertion. Med. Sci. Sport. Exerc. 14, 377-381 (1982).

40. Kendzierski, D. \& DeCarlo, K. J. Physical Activity Enjoyment Scale: Two Validation Studies. J. Sport Exerc. Psychol. 13, 50-64 (2016).

41. Alves, E. D., Panissa, V. L. G., Barros, B. J., Franchini, E. \& Takito, M. Y. Translation, adaptation, and reproducibility of the Physical Activity Enjoyment Scale (PACES) and Feeling Scale to Brazilian Portuguese. Sport Sci. Health 15, 329-336 (2019). 


\section{Acknowledgments}

RABP, VHFL and BMA were supported by the São Paulo Research Foundation (FAPESP) fellowship (\#2019/17445-1, \#2019/22726-0 and \#2019/25282-5, respectively). This study was financed by the São Paulo Research Foundation (FAPESP) (\#2018/21126-6) and in part by the Coordenação de Aperfeiçoamento de Pessoal de Nível Superior - Brasil (CAPES) - Finance Code 001.

\section{Author contribution}

The study was design by de RABP, VHFL, FSL and BMA. RABP and VHFL participated of data acquisition. RABP, VHFL and BMA analyzed the data. RABP, VHFL, FSL, AMZ, AJM and BMA drafted the manuscript. All authors critically reviewed the manuscript, and approved the final manuscript as submitted.

\section{Additional Information}

Competing Interests: The authors declare no conflicts of interest. 
A

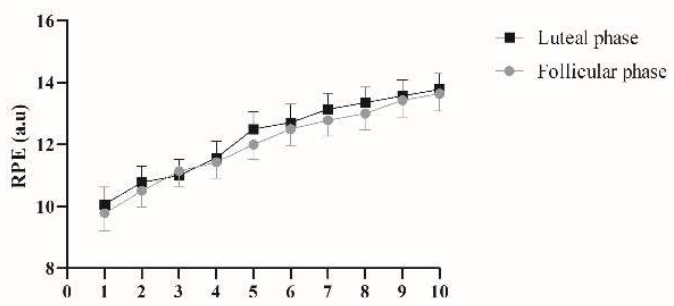

B

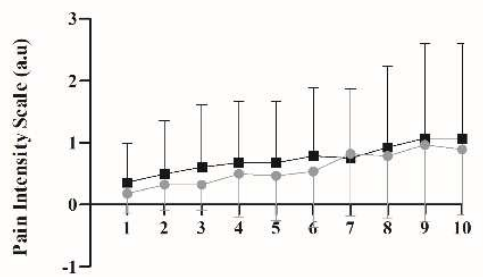

C

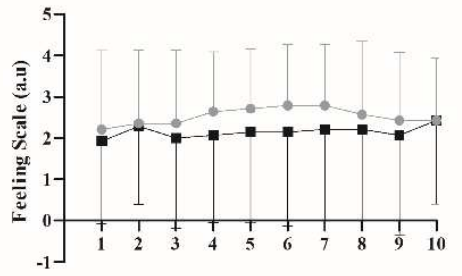

D

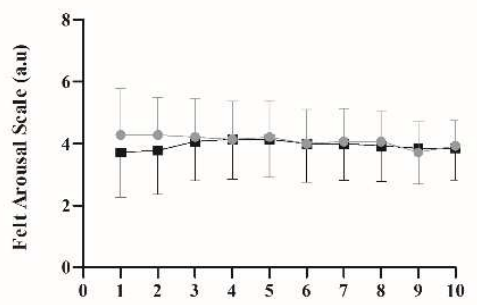

$\mathbf{E}$

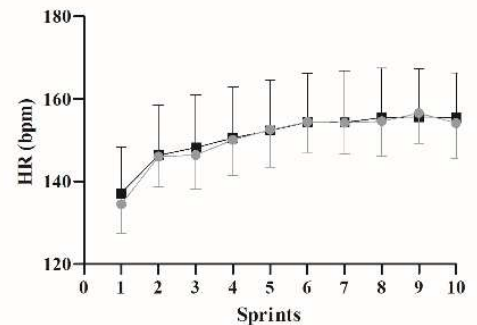

Figure 1. A) Comparison between RPE during HIIE sprints of luteal and follicular phases. B) Comparison between pain during HIIE sprints of luteal and follicular phases. C) Comparison between felling scale during HIIE sprints of luteal and follicular phases. D) Comparison between arousal during HIIE sprints of luteal and follicular phases. E) Comparison between heart rate during HIIE sprints of luteal and follicular phases. 


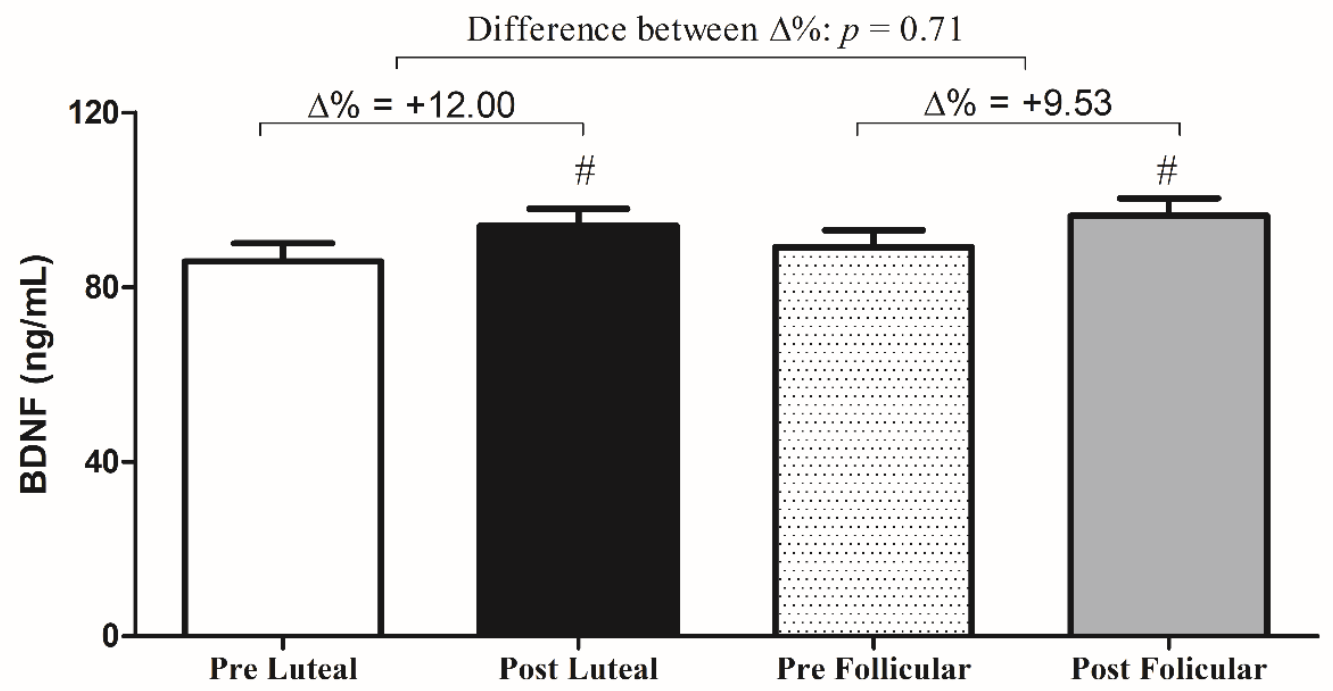

Figure 2. Comparison between BDNF measured pre and post HIIE during luteal and follicular phases; \# Difference between moments (Time effect - ANOVA); * Difference between menstrual phases (Condition effect - ANOVA). 

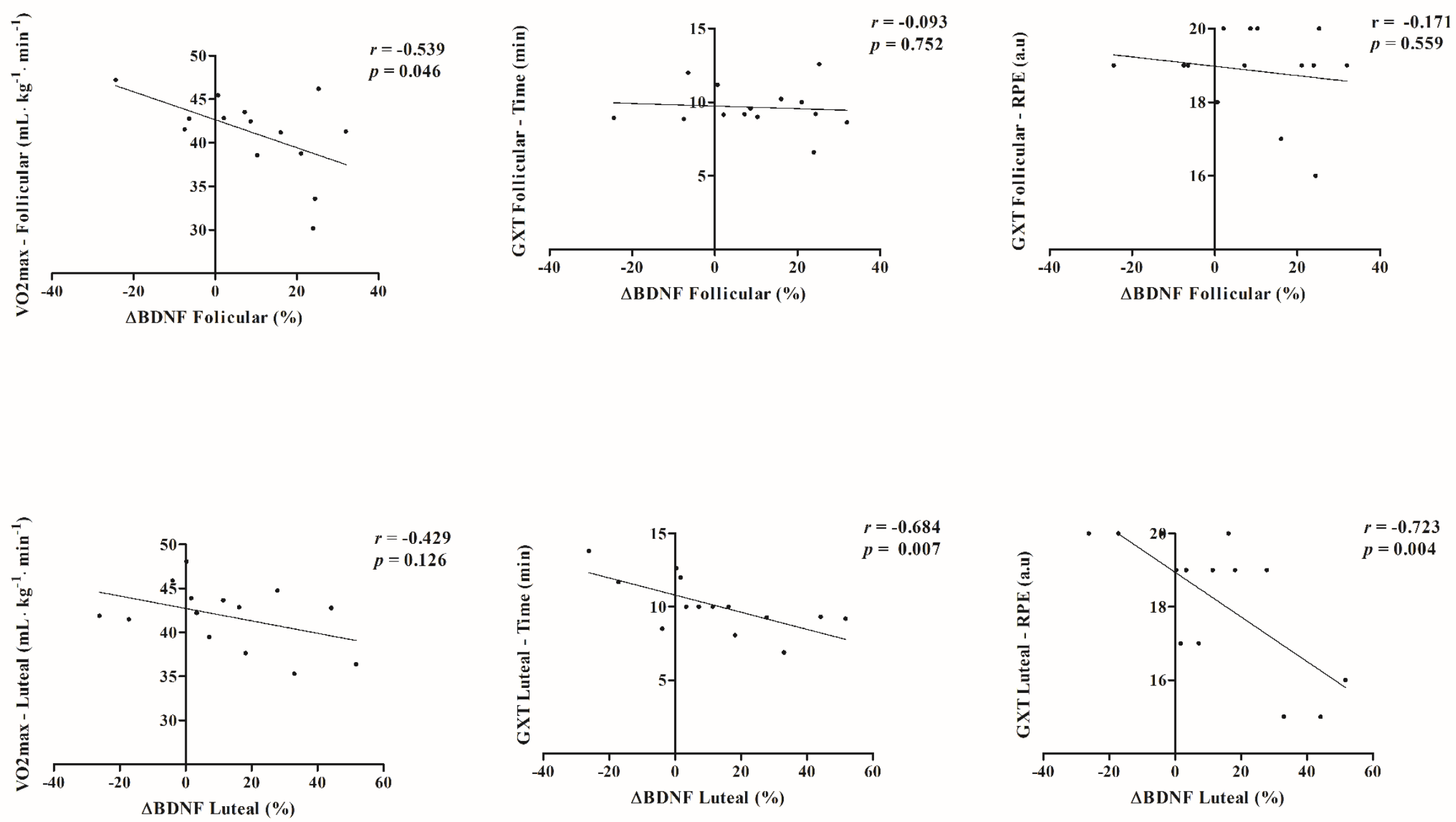

2 Figure 3. Correlation analysis between percentage BDNF changes and GXT variables pre and post HIIE performed at luteal and follicular phases. 
Table 1. Dimensions of mood measured by POMS.

\begin{tabular}{|c|c|c|c|c|c|c|c|}
\hline & \multicolumn{2}{|c|}{ Luteal } & \multicolumn{2}{|c|}{ Follicular } & \multicolumn{3}{|c|}{$\begin{array}{c}\text { ANOVA } \\
p(F)\end{array}$} \\
\hline & Pre & Post & Pre & Post & $\begin{array}{c}\text { Interaction } \\
\text { (time } \mathrm{x} \\
\text { condition) }\end{array}$ & Time effect & $\begin{array}{c}\text { Condition } \\
\text { effect }\end{array}$ \\
\hline Tension \# & $\begin{array}{c}12.77 \pm 6.22 \\
(9.01 \text { to } 16.53)\end{array}$ & $\begin{array}{c}10.54 \pm 6.94 \\
(6.35 \text { to } 14.73)\end{array}$ & $\begin{array}{c}10.54 \pm 2.96 \\
(8.75 \text { to } 12.33)\end{array}$ & $\begin{array}{c}7.38 \pm 4.11 \\
(4.90 \text { to } 9.87)\end{array}$ & $\begin{array}{c}0.323 \\
(1.064)\end{array}$ & $\begin{array}{c}0.003 \\
(14.355)\end{array}$ & $\begin{array}{c}0.052 \\
(4.639)\end{array}$ \\
\hline Depression ${ }^{\#}$ & $\begin{array}{c}8.00 \pm 10.85 \\
(1.44 \text { to } 14.56)\end{array}$ & $\begin{array}{c}5.23 \pm 7.17 \\
(0.90 \text { to } 9.56)\end{array}$ & $\begin{array}{c}4.00 \pm 2.80 \\
(2.31 \text { to } 5.69)\end{array}$ & $\begin{array}{c}2.15 \pm 2.51 \\
(0.64 \text { to } 3.67)\end{array}$ & $\begin{array}{c}0.595 \\
(0.297)\end{array}$ & $\begin{array}{c}0.008 \\
(10.222)\end{array}$ & $\begin{array}{c}0.145 \\
(2.433)\end{array}$ \\
\hline Anger ${ }^{\#}$ & $\begin{array}{c}7.31 \pm 8.06 \\
(2.44 \text { to } 12.18)\end{array}$ & $\begin{array}{c}5.62 \pm 7.19 \\
(1.27 \text { to } 9.96)\end{array}$ & $\begin{array}{c}5.54 \pm 6.37 \\
(1.69 \text { to } 9.39)\end{array}$ & $\begin{array}{c}2.23 \pm 3.79 \\
(-0.06 \text { to } 4.52)\end{array}$ & $\begin{array}{c}0.428 \\
(0.673)\end{array}$ & $\begin{array}{c}0.001 \\
(18.932)\end{array}$ & $\begin{array}{c}0.188 \\
(1.949)\end{array}$ \\
\hline Vigor & $\begin{array}{c}14.46 \pm 7.83 \\
(9.73 \text { to } 19.19)\end{array}$ & $\begin{array}{c}15.69 \pm 8.04 \\
(10.84 \text { to } 10.55)\end{array}$ & $\begin{array}{c}16.92 \pm 6.66 \\
(12.90 \text { to } 20.95)\end{array}$ & $\begin{array}{c}18.23 \pm 6.80 \\
(14.12 \text { to } 22.34)\end{array}$ & $\begin{array}{c}0.954 \\
(0.003)\end{array}$ & $\begin{array}{c}0.197 \\
(1.864)\end{array}$ & $\begin{array}{c}0.310 \\
(5.927)\end{array}$ \\
\hline Fatigue & $\begin{array}{c}8.46 \pm 5.30 \\
(5.26 \text { to } 11.66)\end{array}$ & $\begin{array}{c}7.31 \pm 5.81 \\
(3.80 \text { to } 10.82)\end{array}$ & $\begin{array}{c}5.69 \pm 3.17 \\
(3.78 \text { to } 7.61)\end{array}$ & $\begin{array}{c}5.31 \pm 3.28 \\
(3.33 \text { to } 7.29)\end{array}$ & $\begin{array}{l}0.165 \\
(2.182)\end{array}$ & $\begin{array}{c}0.149 \\
(2.379)\end{array}$ & $\begin{array}{c}0.065 \\
(4.115)\end{array}$ \\
\hline Confusion & $\begin{array}{c}7.31 \pm 4.35 \\
(4.68 \text { to } 9.93)\end{array}$ & $\begin{array}{c}6.15 \pm 4.49 \\
(3.44 \text { to } 8.87)\end{array}$ & $\begin{array}{c}6.31 \pm 4.39 \\
(3.66 \text { to } 8.96)\end{array}$ & $\begin{array}{c}5.08 \pm 4.13 \\
(2.58 \text { to } 7.57)\end{array}$ & $\begin{array}{c}0.856 \\
(0.034)\end{array}$ & $\begin{array}{c}0.092 \\
(3.346)\end{array}$ & $\begin{array}{c}0.826 \\
(0.381)\end{array}$ \\
\hline
\end{tabular}

Values are mean \pm SD (CI95\%). ${ }^{\dagger}$ Difference between time within conditions (Interaction - ANOVA); ${ }^{\#}$ Difference between moments (Time effect - ANOVA); $*$ Difference between menstrual phases (Condition effect - ANOVA) 


\section{Figures}

A

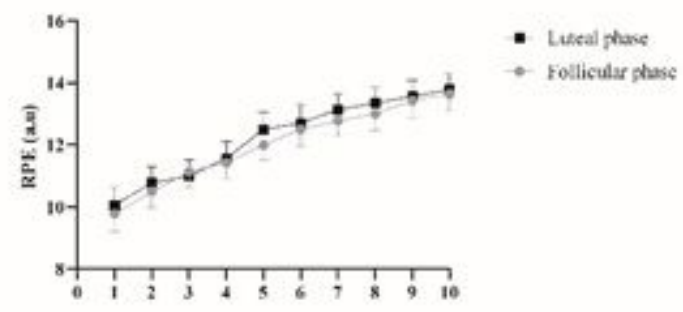

B

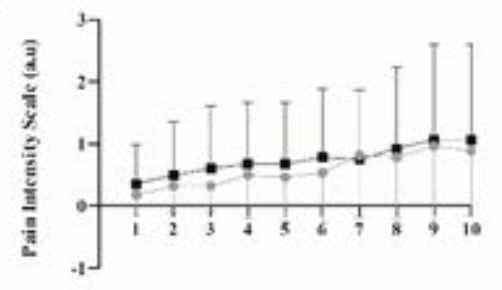

C

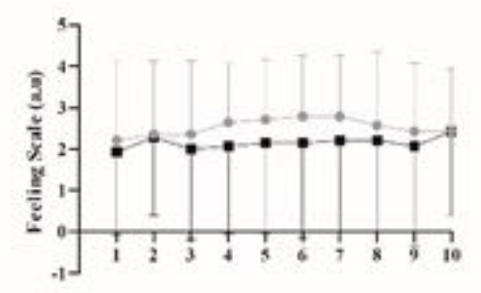

D

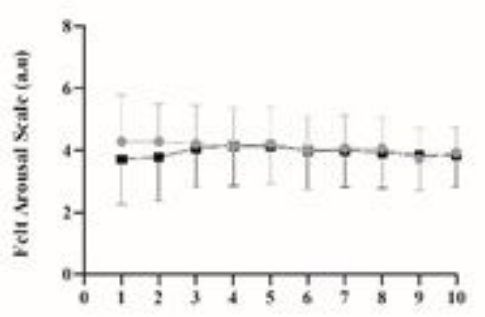

E

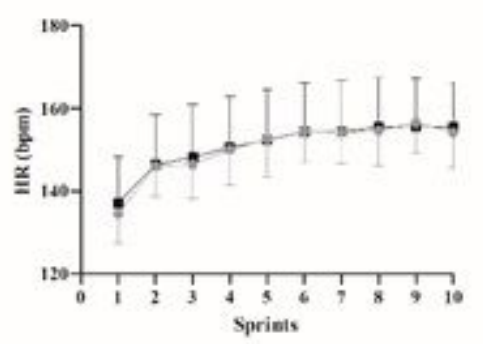

Figure 1

A) Comparison between RPE during HIIE sprints of luteal and follicular phases. B) Comparison between pain during HIIE sprints of luteal and follicular phases. C) Comparison between felling scale during HIIE sprints of luteal and follicular phases. D) Comparison between arousal during HIIE sprints of luteal and follicular phases. E) Comparison between heart rate during HIIE sprints of luteal and follicular phases. 


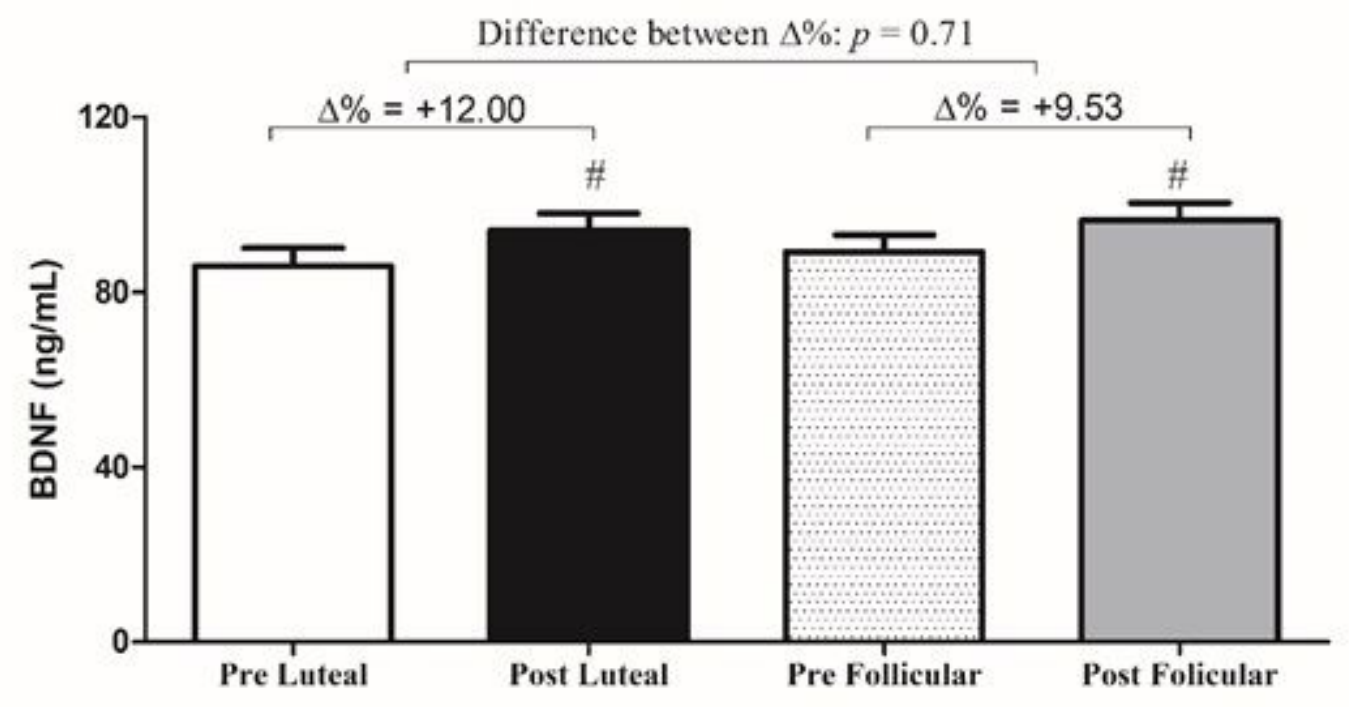

\section{Figure 2}

Comparison between BDNF measured pre and post HIIE during luteal and follicular phases; \# Difference between moments (Time effect - ANOVA); * Difference between menstrual phases (Condition effect ANOVA).
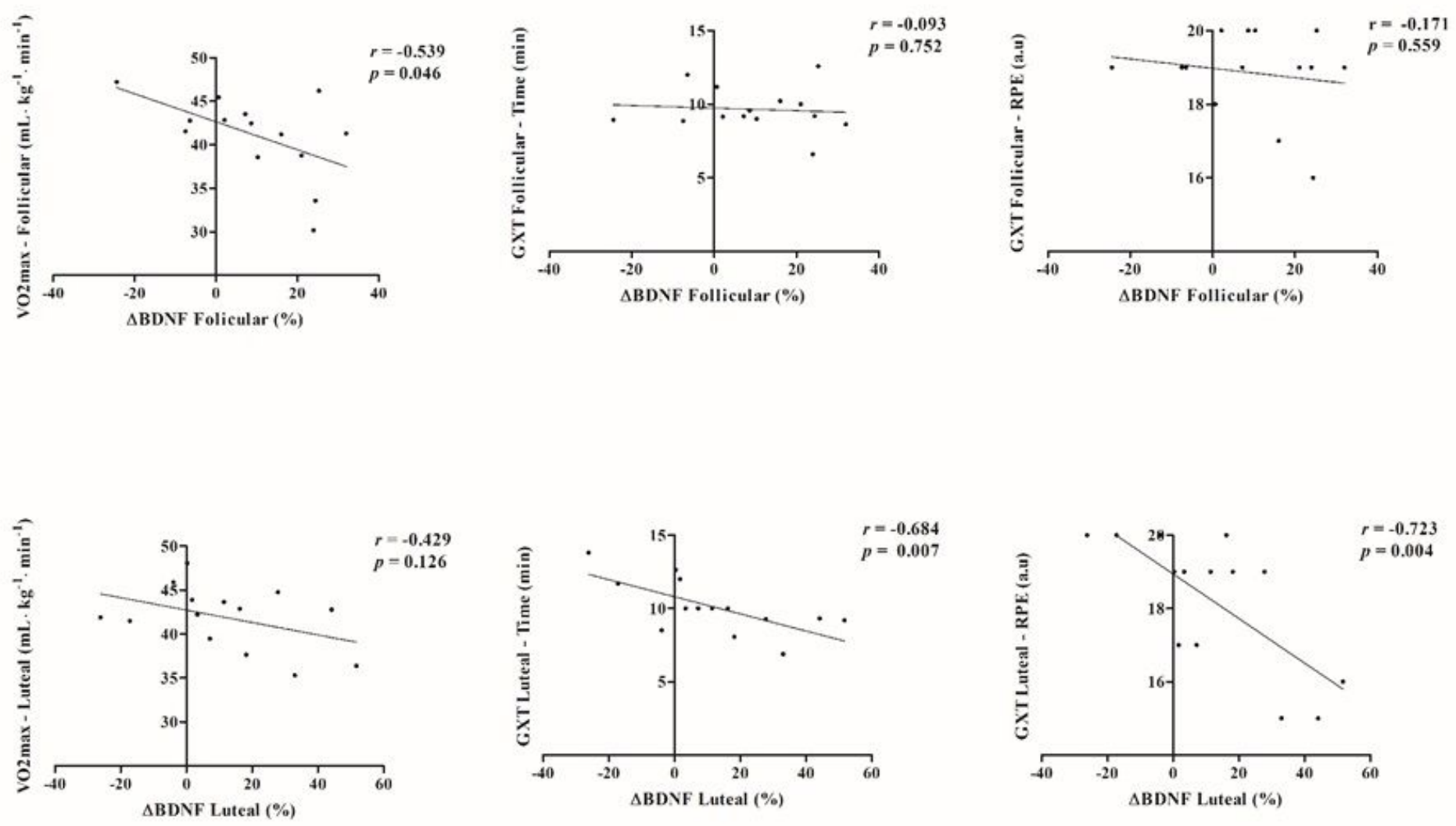

Figure 3 
Correlation analysis between percentage BDNF changes and GXT variables pre and post HIIE performed at luteal and follicular phases.

\section{Supplementary Files}

This is a list of supplementary files associated with this preprint. Click to download.

- Supplementalmaterials.docx 